\title{
Double-Baer groups
}

\author{
Vikram Jha Norman L. Johnson
}

\begin{abstract}
We show that a double-Baer group implies the existence of a doubleretraction in a translation plane with kernel containing a field $K=\mathrm{GF}(q)$. If the associated spread is in $\mathrm{PG}(3, q)$ then a lifted spread in $\mathrm{PG}\left(3, q^{2}\right)$ admits a double-Baer group. The double-retraction group produces a maximal partial mixed partition of $\mathrm{PG}\left(3, q^{2}\right)$ of lines and $\mathrm{PG}(3, q)$. This result is generalized and new examples of translation planes admitting double-Baer groups are given.
\end{abstract}

Keywords: Baer groups, subgeometry partitions, lifting

MSC 2000: primary: 51E23; secondary: 51A40

We both met Gabor Korchmáros in 1985 when the "triangle" of Potenza-BariLecce met for a series of seminars at Lecce and we were visiting the university. Many finite geometers have been greatly influenced by the tremendous depth and range of the geometry of Gabor. In particular, we gratefully acknowledge that our foundations' book on translation planes with Mauro Biliotti grew out of a summer school organized by Gabor and his group at Potenza during the summer of 1996. We are privileged to dedicate this article to Gabor on the occasion of his sixtieth birthday.

\section{Introduction}

This article somewhat concerns the concept of 'geometric lifting', which is described in the text by Hirschfeld and Thas [4]. In particular, from a subgeometry partition of a finite projective space, there is a construction that produces a corresponding finite spread, and hence, from which we obtain a finite translation plane. The subgeometry partition may consist entirely of Baer subgeometries or be a so-called 'mixed subgeometry partition' where the partition has at least two non-isomorphic subgeometries comprising the partition. 


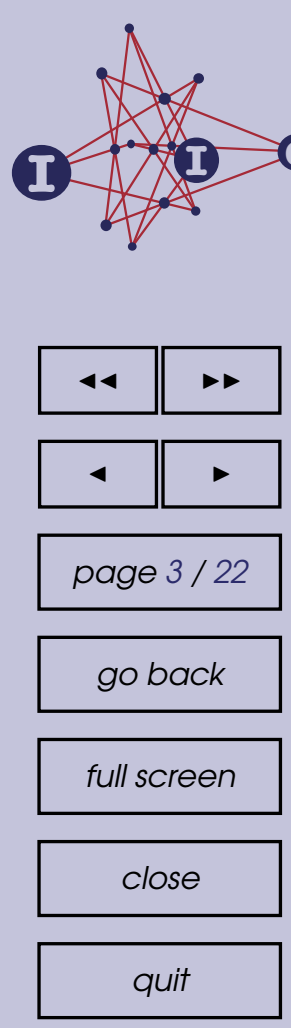

of a given translation plane with the above properties allowing retraction using either one of these fields to produce subgeometry partitions of isomorphic projective spaces.) Furthermore, in Johnson [10] and using Jha and Johnson [5], it is shown that spreads in $\mathrm{PG}\left(3, q^{2}\right)$ lifted from spreads in $\mathrm{PG}(3, q)$ always admit double-Baer groups of the type above and hence there is an associated doubleretraction. In this case, one retraction provides a mixed subgeometry partition of $q^{2}+1$ lines and $q^{2}(q-1) \mathrm{PG}(3, q)$ 's of a PG $\left(3, q^{2}\right)$.

Considered vectorially, retraction may be considered more generally over infinite vector spaces, even infinite vector spaces of infinite dimension over their kernels. Hence, in essence, geometric lifting may be more generally considered, even though in this article, we restrict ourselves to the finite situation.

To be clear on what we mean by 'retraction', we end this section with the second author's theorem involving groups of order $q^{2}-1$ and our specific definitions. The following theorem deals with the situation considered in this article, although the theorem can be stated in a more general form.

Theorem 1.3 (Johnson [10]). Let $\pi$ be a translation plane of order $q^{2^{a} r}$ and kernel containing GF $(q)$. Let $G$ be a collineation group of order $q^{2}-1$ containing the kernel homology group of order $q-1$ and assume that $G \cup\{0\}$ (the zero mapping) is a field $K$.

Then the component orbit lengths of $G$ are either 1 or $q+1$. Forming the projective space $\mathrm{PG}\left(2^{a} r-1, q^{2}\right)$, the orbits of length 1 become projective subgeometries isomorphic to $\mathrm{PG}\left(2^{a-1} r-1, q^{2}\right)$ and the orbits of length $q+1$ become projective subgeometries isomorphic to $\mathrm{PG}\left(2^{a-1} r-1, q\right)$.

The set of subgeometries partition the points of the projective space providing a 'mixed subgeometry partition'.

Furthermore, as mentioned, there is a 'geometric lifting' process that constructs translation planes of order $q^{2^{a} r}$ and kernel containing $\operatorname{GF}(q)$ from any mixed subgeometry partition.

There is also a corresponding result in Johnson [10] for translation planes of order $q^{t}$, for $t$ odd but admitting collineation groups of order $q^{2}-1$ having the properties of the group in the previous theorem. In this setting, all component orbits will have length $q+1$ and the associated subgeometry partition is said to be a 'Baer subgeometry partition'.

In general, we define the process of 'retraction' as follows.

Definition 1.4. Assume that a finite translation plane with kernel containing $\mathrm{GF}(q)$ admits a collineation group of order $q^{2}-1$ that contains the kernel homology group of order $q-1$ such that together with the zero mapping, a field 



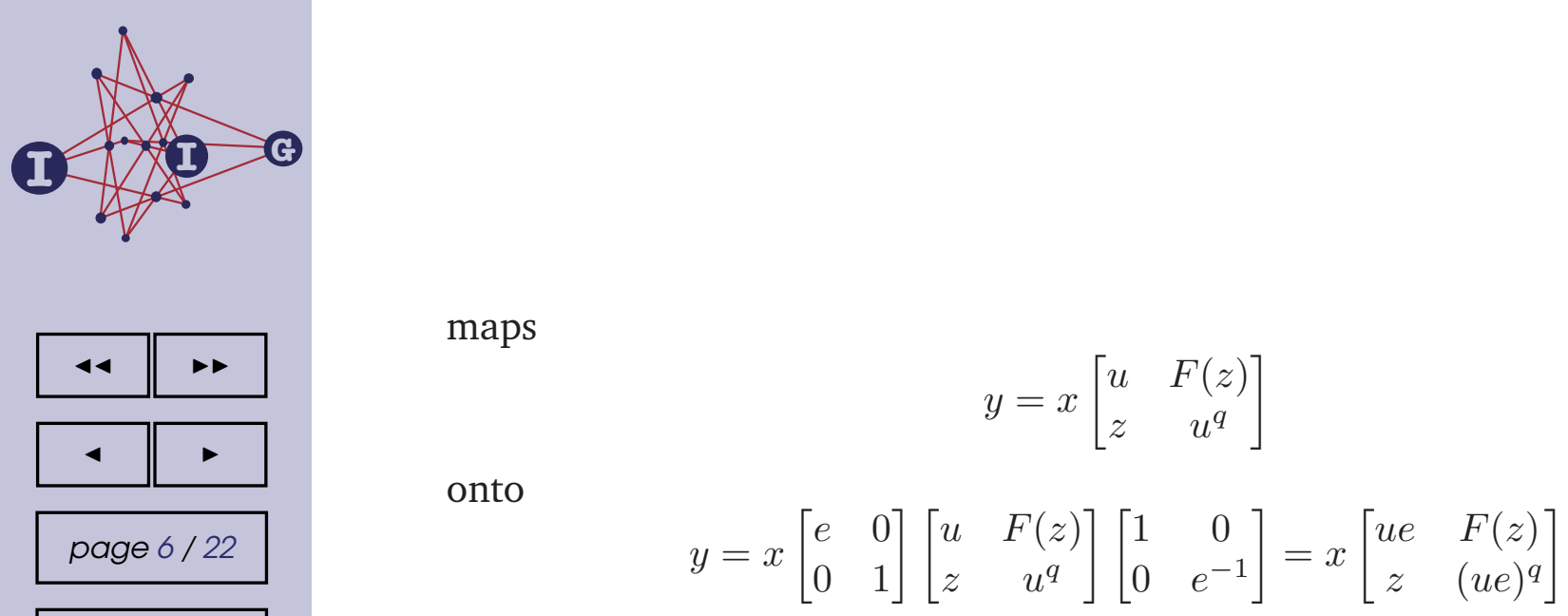

go back

full screen

close

quit

and so is a collineation of the algebraically lifted translation plane. Furthermore, $B_{1}$ fixes the vector subspace

$$
\text { Fix } B_{1}=\left\langle\left(0, x_{2}, y_{1}, 0\right) \mid x_{2}, y_{1} \in \mathrm{GF}\left(q^{2}\right)\right\rangle
$$

pointwise, which implies that $B_{1}$ is a Baer group of order $q+1$. Since, we have kernel GF $\left(q^{2}\right)$ in the lifted plane, it follows that

$$
\left.B_{2}=\langle\operatorname{diag}(1, e, e, 1)| e \text { has order } q+1\right\rangle
$$

is also a Baer group fixing the vector subspace

$$
\left\langle\left(x_{1}, 0,0, y_{2}\right) \mid x_{1}, y_{2} \in \mathrm{GF}\left(q^{2}\right)\right\rangle
$$

pointwise. Furthermore, $B_{1}$ and $B_{2}$ commute and note that $B_{1}$ is contained in the group $B_{2} K^{2 *}$, where $K^{2 *}$ denotes the kernel homology group of order $q^{2}-1$. Now let $K^{2}$ be the kernel homology group of order $q^{2}-1$ and form $\left\langle B_{1}, B_{2}\right\rangle K^{2 *}$, which we claim is

$$
\left.D=\langle\operatorname{diag}(a, b, b, a)| a, b \in \mathrm{GF}\left(q^{2}\right)^{*} \text { such that } a^{q+1}=b^{q+1}\right\rangle
$$

of order $(q+1)\left(q^{2}-1\right)$. Note that the order of $\left\langle B_{1}, B_{2}\right\rangle K^{2 *}$ is $(q+1)^{2}\left(q^{2}-1\right) / I$, where $I$ is the intersection with $B_{1} B_{2}$ and $K^{2 *}$. This group leaves invariant the subplane Fix $B_{1}, B_{1}$ fixes it pointwise, $B_{2}$ induces a kernel group on it as does $K^{2 *}$. Hence, the group induced on Fix $B_{1}$ is isomorphic to $K^{2 *}$. But, since $B_{1}$ of order $q+1$ fixes Fix $B_{1}$ pointwise, it follows that the group has order $(q+1)\left(q^{2}-1\right)$.

When $a=b, D$ contains $K^{2 *}$ and when $a=1, D$ contains $B_{1}$, similarly, $D$ contains $B_{2}$. Since $D$ has a $K^{2 *}$ as a normal subgroup of index $q+1$, we see that $B_{1} B_{2} K^{2 *}=D$.

Let $q=p^{r}$, for $p$ a prime and form the following set of fields $K_{p^{i}}$ of order $q^{2}$, for $\sigma=p^{i}$ for $i$ dividing $2 r$.

$$
L_{\sigma}=\left\langle\operatorname{diag}\left(a, a^{\sigma}, a^{\sigma}, a\right) \mid a \in \mathrm{GF}\left(q^{2}\right)^{*}\right\rangle \cup\{0\} .
$$

To obtain $L_{\sigma}^{*}$ in our group, then $a^{\sigma(q+1)}=a^{q+1}$, which implies that if $\sigma=p^{i}$, then $q$ divides $p^{i}$, so let $\sigma=q$ or 1 . 


$(2, q-1)(q+1)$. Now if we reread the argument given in the previous subsection, we see that we can still create two fields $L_{\sigma}$, for $\sigma=1$ or $q$. In particular, we have

$$
\begin{aligned}
& B_{1} \operatorname{co} B_{1} K_{q}^{*}=\langle\operatorname{diag}(C \alpha, A \alpha, C \alpha, A \alpha)| C \text { and } A \text { have } \\
& \left.\quad \text { orders divisible by }(2, q-1)(q+1) z \text { and } \alpha \in K_{q}^{*}\right\rangle .
\end{aligned}
$$

The order of this group is $((2, q-1)(q+1))^{2}(q-1) / I$, where $I$ is the intersection of $B_{1} \operatorname{co} B_{1}$ and $K_{q}^{*}$. In order that an element of $g \in B_{1} \operatorname{co} B_{1}$ be also in $K_{q}^{*}$, then $C=A$ has order dividing $(q-1)$. If $q$ is even, then $I$ is clearly $\langle 1\rangle$, so assume that $q$ is odd. Then, we have a group of order $4(q+1)^{2}(q-1) / I$. First assume that $(q-1) / 2$ is odd. Then clearly we have a group of order $2(q+1)\left(q^{2}-1\right)$. If $(q-1) / 2$ is even then there is a cyclic group of order 4 in both $B_{1}, \operatorname{co} B_{1}$ and $K_{q}^{*}$, so that the group has order $(q+1)\left(q^{2}-1\right)$.

Therefore, in all situations, we have the subgroup of $B_{1} \operatorname{co} B_{1} K_{q}^{*}$

$$
\left.\langle\operatorname{diag}(D, E, D, E)| D^{q+1}=E^{q+1}, \text { for } D \in K_{1}^{+}, E \in K_{2}^{*}\right\rangle
$$

of order $(q+1)\left(q^{2}-1\right)$ and we obtain the fields $L_{\sigma}$, for $\sigma=1$ or $q$, exactly as in the previous situation.

However, in this setting, the field $L_{1}$ need not be a kernel homology group. Therefore, we obtain the following theorem.

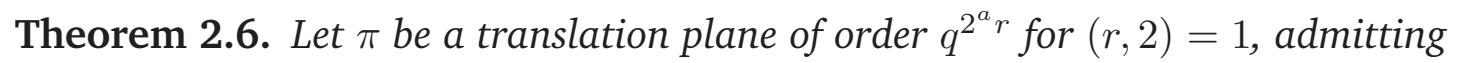
a double-Baer group of of order divisible by $(2, q-1)(q+1)$ with kernel containing $K_{q}$ isomorphic to $\mathrm{GF}(q)$.

(1) From the double-Baer group, we may construct two retraction fields $L_{\sigma}$, for $\sigma=1$ or $q$, for the translation plane so we have double-retraction.

(2) If the plane has kernel $\mathrm{GF}\left(q^{2}\right)$ but $L_{1}$ is not a kernel homology group, we obtain triple-retraction.

\section{The fusion of Baer groups}

One important procedure for the construction of double-Baer groups is the following theorem.

Theorem 3.1. Let $\pi$ be a semifield plane of order ${q^{2^{a}} r}$ and kernel containing $K$ isomorphic to $\mathrm{GF}(q)$, for $(r, 2)=1$. Assume that the right nucleus and middle nucleus of the associated semifield contain fields isomorphic to $\operatorname{GF}\left(q^{2}\right)$. If there is a derivable net that contains a middle nucleus net invariant under the associated affine homology group then the derived plane admits a double-Baer group. 




with fixed point space

$$
\text { Fix } B_{1}=\left\{\left(x_{1}, 0, x_{3}, 0,0, x_{6}\right) \mid x_{1}, x_{3}, x_{6} \in \mathrm{GF}\left(q^{2}\right)\right\} .
$$

In the second group, we have the Baer group

$$
B_{2}=\left\langle\operatorname{diag}\left(1, a^{q-1}, 1, a^{1-q}, 1, a^{1-q}\right) \mid a \in \mathrm{GF}\left(q^{2}\right)^{*}\right\rangle
$$

with fixed point space

$$
\text { Fix } B_{2}=\left\{\left(x_{1}, 0, x_{3}, 0, x_{5}, 0\right) \mid x_{1}, x_{3}, x_{5} \in \mathrm{GF}\left(q^{2}\right)\right\} \text {. }
$$

Hence, $B_{1} B_{2}$ fixes

$$
\text { Fix } B_{1} \cap \text { Fix } B_{2}=\left\{\left(x_{1}, 0, x_{3}, 0,0,0\right) \mid x_{1}, x_{3}, x_{5} \in \mathrm{GF}\left(q^{2}\right)\right\} \text {, }
$$

which cannot be a subplane or line or either, which is a contradiction. In a similar manner and in general, it follows fairly direct that if we have $\left(2^{n}-1\right)$ retraction in a translation plane of order $q^{2 n}$, then $n \leq 2$ and since retraction makes sense only when $n \geq 2$, we have the following theorem.

Theorem 3.8. Let $\pi$ be a translation plane of order $q^{2 n}$ and kernel containing $\mathrm{GF}(q)$ that admits $\left(2^{n}-1\right)$-retraction. Then, $n=2$ and we obtain triple-retraction.

\section{Double-homology groups}

We revisit part of our previous results and show connections to what are called 'double-homology groups'.

Theorem 4.1. Let $\pi$ be a translation plane of order $q^{2^{a} r}$ and kernel containing $K$ isomorphic to $\mathrm{GF}(q)$.

(1) When $q$ is even, a double-Baer group of order $q+1$ implies double-retraction.

(2) For arbitrary order, a double-Baer group of order $q+1$ with intertwining field implies double-retraction.

(3) A double-Baer group of order $(2, q-1)(q+1)$ implies double-retraction.

(4) Double-retraction implies a double-Baer group of order $q+1$ or two commuting homology groups of order $q+1-a$ 'double-homology group' of order $q+1$.

(5) A double-homology group of order $q+1$ in a semifield plane implies doubleretraction.

Proof. It remains to prove (4) and (5). Assume that we have double-retraction. Write one of the fixed-point-free groups as $G_{1} K^{*}$ and decompose the space so 




[11] N. L. Johnson and K. E. Mellinger, Multiple spread retraction, Adv. Geom. 3 (2003), no. 3, 263-286.

\author{
Vikram Jha \\ Mathematics Dept., University of IowA, Iowa City, Iowa 52242, USA \\ e-mail: vjha267@googlemail.com \\ Norman L. Johnson \\ Mathematics Dept., University of IowA, IowA City, Iowa 52242, USA \\ e-mail: njohnson@math.uiowa.edu

quit 